\title{
A microscope using Zernike's phase contrast method and a hard x-ray Gabor hologram
}

\author{
Kiyofumi Matsuda ${ }^{1,2}$, Juan C. Aguilar ${ }^{3}$, Masaki Misawa ${ }^{1 *} \mathbb{D}$, Masato Yasumoto ${ }^{4,5}$, Shakil Rehman ${ }^{6}$, Yoshio Suzuki ${ }^{5}$, \\ Akihisa Takeuchi ${ }^{5}$ and IIpo Niskanen ${ }^{7}$
}

\begin{abstract}
Background: In hard X-ray phase imaging using interferometry, the spatial resolution is limited by the pixel size of digital sensors, inhibiting its use in magnifying observation of a sample.

Methods: To solve this problem, we describe a digital phase contrast microscope that uses Zernike's phase contrast method with a hard X-ray Gabor holography associated with numerical processing and spatial frequency domain filtering techniques. The hologram is reconstructed by a collimated beam in a computer. The hologram intensity distributions itself become the reconstructed wavefronts. For this transformation, the Rayleigh- Sommerfeld diffraction formula is used.

Results: The hard X-ray wavelength $0.1259 \mathrm{~nm}$ (an energy of $9.85 \mathrm{keV}$ ) was employed at the SPring-8 facility. We succeeded in obtaining high-resolution images by a CCD sensor with a pixel size of $3.14 \mu \mathrm{m}$, even while bound by the need to satisfy the sampling theorem and by the CCD pixel size. The test samples used here were polystyrene beads of 8 $\mu \mathrm{m}$, and human HeLa cells.

Conclusions: We thus proved that the resolution $0.951 \mu \mathrm{m}$ smaller than the pixel size of CCD $(3.14 \mu \mathrm{m})$ was achieved by the proposed reconstruction techniques and coherent image processing in the computer, suggesting even higher resolutions by adopting greater numerical apertures.
\end{abstract}

Keywords: X-ray microscopy, Distributed-feedback, Digital holography, X-ray imaging, Interference microscopy, X-ray interferometry

\section{Background}

Hard X-rays allow visualization of objects at high resolutions because of their inherent smaller wavelengths [1]; they are therefore highly desirable in various application areas, including biological studies [2, 3]. Even though optical imaging methods are non-destructive (and therefore useful), they are typically limited in spatial resolution because of the long wavelengths used [4] and transmission limitations. Imaging with electron beams depends on fixing the sample under vacuum conditions, and this is detrimental to the morphology of the biological specimens under observation. On the other hand, hard X-rays may suffer the effect of external vibrations, because of their short wavelengths.

Phase imaging using hard X-ray makes it possible to visualize phase objects using interferometry or holography.

\footnotetext{
* Correspondence: m.misawa@aist.go.jp

${ }^{1}$ Theranostic Device Research Group, Health Research Institute, National Institute of Advanced Industrial Science and Technology (AIST), 1-2-1 Namiki, Tsukuba, Ibaraki 305-8564, Japan

Full list of author information is available at the end of the article
}

Computed tomography using an X-ray interferometer [5] and observation of biological soft tissues using interferometry [6] have been used for phase imaging. Methods using differential interference contrast (DIC) to form Talbot images with hard X-rays have been reported [6]. Given that samples are imaged on a digital sensor, the spatial resolution in such methods is limited, because of the pixel size.

Many reports of digital holography [7] and holographic lateral shear for DIC [8] using laser beam holography have been made. High resolution is possible in holography, because the images are obtained at a position apart from the digital sensor. The two-point resolution in coherent illumination hard X-rays can be obtained by $0.82 \lambda / N A$ [9]-if the relative phase between the two points can be considered as zero-where $\lambda$ is the wavelength and $N A$ is the numerical aperture. It should be pointed out that the sensor pixel size does not appear in the equation for resolution. Accordingly, hard X-ray digital holographic systems may provide high resolution. However, the conjugate images 
and reconstructed images overlap, a problem that must be solved. Hard X-rays have been used in Gabor holography [10], to obtain speckle-free coherent illumination [1-11], and in phase contrast microscopy [12]. Hard X-rays have also been used in coherent diffractive imaging [13] with high sensitivity, lens-less imaging with an extended source [14], and phase contrast imaging [15] —using polychromatic hard X-rays [16].

This paper describes a digital holographic microscope using Zernike's phase contrast observation method [4] using a hard X-ray Gabor hologram, which is recorded in a computer. In Gabor holograms, the most important aspect is to reduce the effect of conjugate images. Good quality high-resolution images were obtained after some numerical processing.

\section{Methods}

Description of the used hard X-ray Gabor hologram setup Figure 1 shows the Gabor hologram process using a common path interferometer. In our optical arrangement, the maximum optical path difference between the reference and object beams in the hologram plane is very short. The synchrotron source (the beamline 20XU of SPring-8, Japan) used in this study produces quasi-monochromatic ${ }^{4} \mathrm{X}$-rays with a monochromator, as shown in Fig. 1. The central part of the expanded X-ray beam is filtered by aperture $\mathrm{CS}_{1}$, with a size of $50 \mu \mathrm{m}$, and is focused by a Fresnel zone plate (FZP) with a diameter of $104 \mu \mathrm{m}$ and a focal length of $498 \mathrm{~mm}$. Another cross slit aperture $\mathrm{CS}_{2}$, with a size of $2 \mu \mathrm{m}$, is used to filter the beam and acts as the point source for hologram recording. The beam illuminates an object placed at a distance of $Z_{0}$ from $\mathrm{CS}_{2}$. The size of the hologram is determined by the size of the reference beam at the hologram plane (diameter $\mathrm{D}=1.483 \mathrm{~mm}$ at a distance of $Z_{1}$ from $\mathrm{CS}_{2}$ ). The distance between the object and the hologram is $Z$. A 16-bit Hamamatsu charge-coupled device (CCD) sensor (C4742-98-24) with a $3.14 \mu \mathrm{m}$ pixel size which consists of $1344 \times 1024$ pixels was used to record the holograms. Interference fringes with a narrowest spacing of $15.3 \mu \mathrm{m}$ were produced at the edges of the sensor. The minimum width of the fringe was about 4.9 times the pixel size, thus satisfying the sampling theorem. The X-ray energy was 9.85 $\mathrm{keV}$, with a corresponding wavelength of $0.1259 \mathrm{~nm}$. The test samples used in this study were $8 \mu \mathrm{m}$ polystyrene sphere beads and human HeLa cells. The spatial resolution of the reconstructed image in our setup was calculated at $0.951 \mu \mathrm{m}[1]$, which is less than the sensor pixel size.

The Gabor hologram is reconstructed by numerical processing in the computer. In the conventional reconstruction method, the object image and its twin image appear close to each other, as shown in Fig. 2, and removal of this twin image is therefore crucial to obtain the final image [17]. To reduce the effects of both the dc noise and the twin image, defocus is introduced in the image plane. For reconstruction, a collimated beam parallel to the optical axis is used to illuminate the hologram, so that the complex amplitude of the reconstructed image is obtained by simply carrying out Fresnel back propagation of the hologram to the image plane. In this way, the twin image is produced far from the object image, as shown in Fig. 3. The convolution of the twin image with an impulse response having a diverging curvature produces a defocus, thereby reducing the twin image effects.

\section{Mathematical description of Zernike's method}

The Zernike's phase contrast method can be mathematically derived using the complex amplitudes reconstructed from the Gabor hologram, as will be shown. If the complex amplitudes of the reference beam and the phase object beam in the hologram plane at coordinates $\left(x_{1}, y_{1}\right)$ are denoted by $r\left(x_{1}, y_{1}\right)$ and $g\left(x_{1}, y_{1}\right)$ respectively, the intensity distribution $I_{\mathrm{h}}\left(x_{1}, y_{1}\right)$ of hologram is given by

$$
\begin{aligned}
I_{h}\left(x_{1}, y_{1}\right)= & \left|r\left(x_{1}, y_{1}\right)+g\left(x_{1}, y_{1}\right)\right|^{2} \\
= & {\left[\left|r\left(x_{1}, y_{1}\right)\right|^{2}+\left|g\left(x_{1}, y_{1}\right)\right|^{2}\right]+r^{*}\left(x_{1}, y_{1}\right) g\left(x_{1}, y_{1}\right) } \\
& +r\left(x_{1}, y_{1}\right) g^{*}\left(x_{1}, y_{1}\right)
\end{aligned}
$$

The complex amplitude of $r\left(x_{1}, y_{1}\right)$ is given by

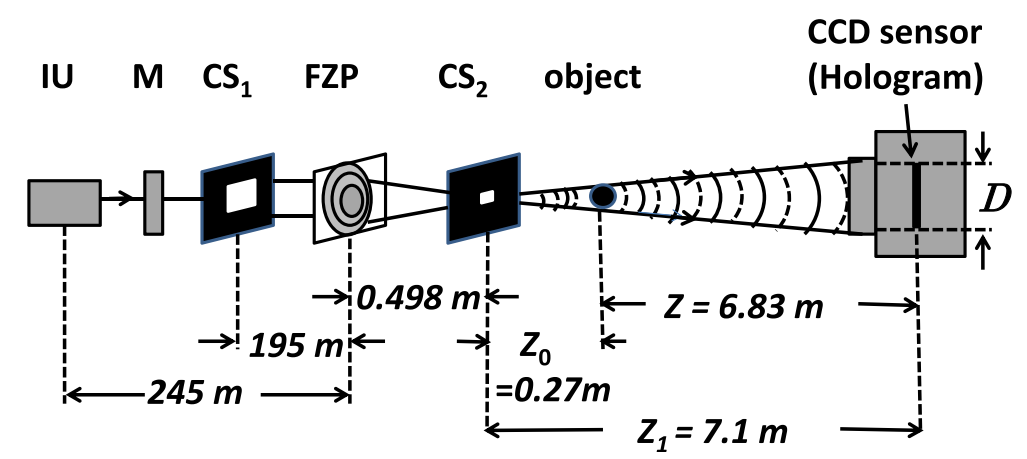

Fig. 1 Optical arrangement for the recording of a hard X-ray Gabor hologram. IU is an in-vacuum undulator, $M$ a monochromatic meter, $\mathrm{CS}_{1}$ a crossslit aperture for a pseudo-point source of size $50 \times 50 \mu \mathrm{m}, \mathrm{CS}_{2}$ a cross-slit aperture for spatial filtering of size $2 \times 2 \mu \mathrm{m}$, and FZP a Fresnel zone plate 


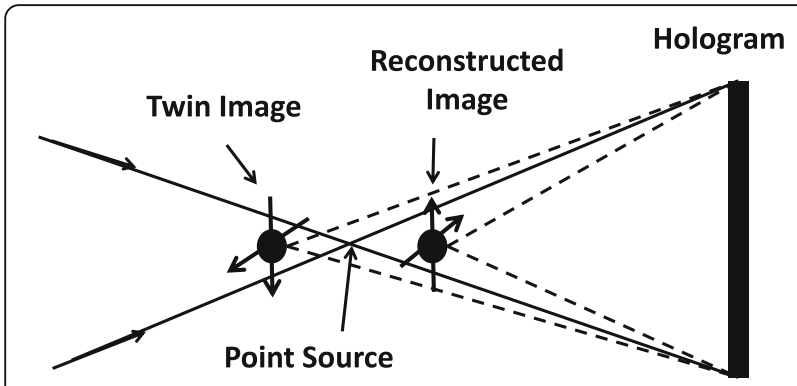

Fig. 2 Optical reconstruction scheme, showing the positions of the image and its twin image

$$
r\left(x_{1}, y_{1}\right)=\left(\frac{1}{j \lambda Z_{1}}\right) e^{\frac{j 2 \pi Z_{1}}{\lambda}} e^{\frac{j \pi}{\lambda Z_{1}}\left(x_{1}^{2}+y_{1}^{2}\right)}
$$

where $Z_{1}$ is the distance between the point source and the hologram, and $\lambda$ is the $\mathrm{X}$-ray wavelength, and $j$ is an imaginary unit, $j=\sqrt{-1}$. If the phase object is illuminated by the hard X-rays originating from a point source, the complex amplitude $\mathrm{g}\left(x_{1}, y_{1}\right)$ of the phase object in the hologram plane is given by

$$
\begin{aligned}
g\left(x_{1}, y_{1}\right)= & -\frac{1}{\lambda^{2} Z_{0} Z} e^{\frac{j 2 \pi\left(Z_{0}+Z\right)}{\lambda}} e^{\frac{j \pi}{\lambda Z}\left(x_{1}{ }^{2}+y_{1}{ }^{2}\right)} \\
& \times \iint e^{\frac{j \pi}{\lambda}\left(\frac{1}{Z_{0}}+\frac{1}{Z}\right)\left(x^{2}+y^{2}\right)-j \Phi(x, y)} e^{-j \frac{2 \pi}{\lambda Z}\left(x_{1} x+y_{1} y\right)} d x d y,
\end{aligned}
$$

where $Z_{0}$ is the distance between the point source and the object, $Z$ is the distance between the object and the hologram, and $\Phi(x, y)$ is the phase distribution of the object. It is noted that if the phase object is thicker, the wavefront is further advanced. The intensity distribution $I_{\mathrm{h}}\left(x_{1}, y_{1}\right)$ of the hologram is obtained by substituting Eq. (2) and Eq. (3) into Eq. (1).

For reconstruction the Gabor hologram is illuminated by a collimated beam of unity amplitude to separate the

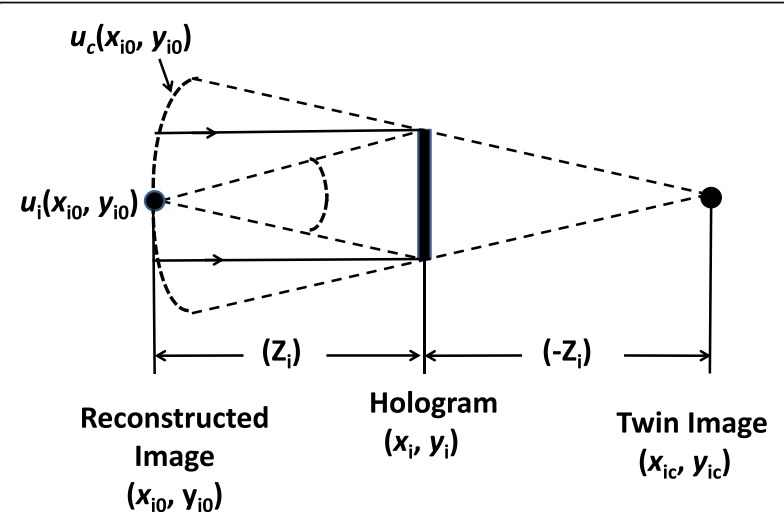

Fig. 3 Positions of the image and its twin image based on the numerical reconstruction method object image and its conjugate image. The complex amplitudes reconstructed in the image plane are obtained by carrying out a Fresnel Transform of $I_{\mathrm{h}}\left(x_{1}, y_{1}\right)$ from the hologram plane to the image plane. The complex amplitude of image $u_{\mathrm{i}}\left(\mathrm{x}_{\mathrm{i}}, \mathrm{y}_{\mathrm{i}}\right)=\operatorname{Fr}\left[r^{\prime \prime}\left(x_{1}, y_{1}\right) g\left(x_{1}, y_{1}\right)\right]$ is given by

$$
\begin{aligned}
& u_{i}(x, y) \\
& =\frac{1}{j \lambda Z_{i}} e^{j 2 \pi Z_{i}} \iint r^{*}\left(x_{1}, y_{1}\right) g\left(x_{1}, y_{1}\right) e^{\frac{j \pi}{\lambda Z_{i}}\left[\left(x_{i}-x_{1}\right)^{2}+\left(y_{i}-y_{1}\right)^{2}\right]} d x_{1} d y_{1} \\
& =-\frac{1}{\lambda^{4} Z_{0} Z Z_{1} Z_{i}} e^{\frac{j 2 \pi Z_{i}}{\lambda}} e^{\frac{j \pi}{\lambda Z_{i}}\left(x_{i}{ }^{2}+y_{i}{ }^{2}\right)} \iint e^{\frac{j \pi}{\lambda}\left(\frac{1}{Z_{0}}+\frac{1}{Z}\right)\left(x^{2}+y^{2}\right)-j \Phi(x, y)} \\
& \times\left\{\begin{array}{c}
\iint e^{\frac{j \pi}{\lambda}\left(\frac{1}{Z}-\frac{1}{Z_{1}}+\frac{1}{Z i}\right)\left(x_{1}{ }^{2}+y_{1}{ }^{2}\right)} \\
e^{-\frac{j 2 \pi}{\lambda}\left[\left(\frac{x}{Z}+\frac{x_{i}}{Z_{i}}\right) x_{1}+\left(\frac{x}{Z}+\frac{x_{i}}{Z_{i}}\right) y_{1}\right]_{d x_{1} d y_{1}}}
\end{array}\right\} d x d y .
\end{aligned}
$$

The image is reconstructed at position $Z_{\mathrm{i} 0}$ where the condition $1 / Z-1 / Z_{1}+1 / Z_{\mathrm{i} 0}=0$ is satisfied; the image plane position is therefore given by $Z_{\mathrm{i} 0}=-Z Z_{1} / Z_{0}$ (using $Z_{1}=Z_{0}+Z$ ). The complex amplitude of the reconstructed image is given by

$$
u_{i}\left(x_{i 0}, y_{i 0}\right)=\frac{1}{\lambda^{2} Z_{1}{ }^{2}} e^{\frac{-j 2 \pi}{\lambda} Z_{i 0}} e^{\frac{j \pi}{\lambda}\left(\frac{1}{Z_{i 0}}+\frac{1}{M^{2} Z_{0}}+\frac{1}{M^{2} Z}\right)\left(x_{i 0}{ }^{2}+y_{i 0}{ }^{2}\right)} e^{-j \Phi\left(\frac{x_{i 0}}{\frac{M, y_{i 0}}{M}}\right)},
$$

where $\Phi\left(x_{\mathrm{i} 0} / M, y_{\mathrm{i} 0} / M\right)$ is a real periodic function (with period $d)$ and $M=\left|Z_{\mathrm{i} 0} / Z\right|$ is the system magnification. We assume that the magnitude of $\Phi\left(x_{\mathrm{i} 0} / M, y_{\mathrm{i} 0} / M\right)$ is small compared to unity, so that we may write [4]

$$
\begin{aligned}
u_{i}\left(x_{i 0}, y_{i 0}\right) & \\
= & \frac{1}{\lambda^{2} Z_{1}^{2}} e^{\frac{-j 2 \pi Z_{i 0}}{\lambda}} e^{\frac{j \pi}{\lambda}\left(\frac{1}{2 Z_{i 0}}\right)\left(x_{i 0}{ }^{2}+y_{i 0}{ }^{2}\right)} e^{\frac{j \pi}{\lambda}\left(\frac{1}{2 Z_{i 0}}+\frac{1}{M^{2} Z_{0}}+\frac{1}{M^{2} Z}\right)\left(x_{i 0}{ }^{2}+y_{i 0}{ }^{2}\right)} \\
& \times\left[1-j \Phi\left(\frac{x_{i 0}}{\frac{M, y_{i 0}}{M}}\right)\right]
\end{aligned}
$$

where the amplitude $k_{\mathrm{i}}=1 /\left(\lambda^{2} Z_{1}^{2}\right)$ is constant, and $\operatorname{Fr}[]$ stands for the Fresnel transform. $Z_{\mathrm{i} 0}=-Z Z_{1} / Z_{0}$ is thus obtained. It is also noted that $\exp [\mathrm{j} \Phi]=1+$ $\mathrm{j} \Phi-\Phi^{2} / 2 !+\cdots$.

Similarly, we can derive the complex amplitude of the conjugate image $u_{\mathrm{c}}\left(x_{\mathrm{i}}, y_{\mathrm{i}}\right)$ in the plane where the conjugate image is reconstructed; $u_{\mathrm{c}}\left(x_{\mathrm{i}}, y_{\mathrm{i}}\right)$ is then given by

If the conjugate image is reconstructed at position $Z_{\mathrm{i}}$ $=Z_{\mathrm{ic}}$, the condition $1 / Z-1 / Z_{1}-1 / Z_{\mathrm{ic}}=0$ must be satisfied. The position in the conjugate image plane $\left(x_{\mathrm{ic}}, y_{\mathrm{ic}}\right)$ is $Z_{\text {ic }}=Z Z_{1} / Z_{0}$, and the complex amplitude of conjugate image is given by 


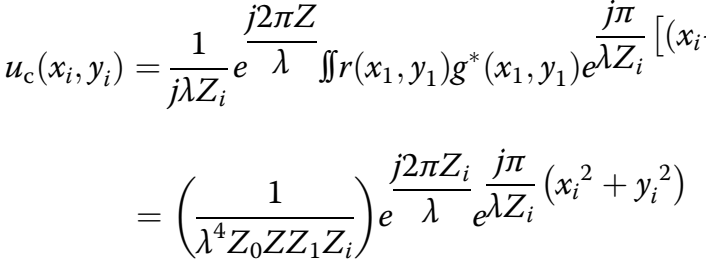

$$
\begin{aligned}
& \times \iint_{e}^{\frac{-j \pi}{\lambda}}\left(\frac{1}{Z_{0}}+\frac{1}{Z}\right)\left(x^{2}+y^{2}\right)+j \Phi(x, y) \\
& u_{\mathrm{c}}\left(x_{i c}, y_{i c}\right) \\
& =\frac{Z}{\lambda^{2} Z_{0} Z_{1} Z_{i c}} e^{\frac{j 2 \pi}{\lambda} Z_{i c}} e^{\frac{-j \pi}{\lambda Z_{i c}}}\left(x_{i c}{ }^{2}+y_{i c}{ }^{2}\right) \\
& e^{-\frac{j \pi}{\lambda}\left(\frac{1}{Z_{0}}+\frac{1}{Z}\right)\left(\frac{Z}{Z_{i c}}\right)^{2}\left(x_{i c}{ }^{2}+y_{i c}{ }^{2}\right)+j \Phi\left(\frac{Z}{Z_{i c}} x_{i c}, \frac{Z}{Z_{i c}} y_{i c}\right)} .
\end{aligned}
$$

If the distance between the reconstructed image and its conjugate image is very long, a wavefront coming from the conjugate image plane may be considered as being emanated from a point source. This condition will be met if the radius $M D / 2$ of the aperture in the image plane is smaller than the Airy disc caused by its aperture at the conjugate image plane; that is, the coherent illumination condition is given by

$$
D^{2}<\frac{4\left(0.82 \lambda Z_{i c}\right)}{M}
$$

It is noted that the magnitudes at the image plane and conjugate image plane are equal. We shall now calculate the complex amplitude of the conjugate image in the image plane caused by a point in the conjugate image plane. In Eq. (8), $x_{\mathrm{ic}}=y_{\mathrm{ic}}=0$ is substituted and given by

$$
u_{\mathrm{c}}\left(x_{i c}, y_{i c}\right)=\frac{Z}{\lambda^{2} Z_{0} Z_{1} Z_{i c}} e^{\frac{j 2 \pi}{\lambda} Z_{i c}} e^{j \Phi(0,0)}
$$

In the image plane $\left(x_{\mathrm{i} 0}, y_{\mathrm{i} 0}\right)$, the complex amplitude of conjugate image is given by

$$
u_{\mathrm{c}}\left(x_{i 0}, y_{i 0}\right)=-\frac{j}{2 \lambda^{3} Z_{1}^{2} Z_{i c}} e^{-j \frac{2 \pi}{\lambda} Z_{i c}} e^{-\frac{j \pi}{2 \lambda Z_{i c}}}\left(x_{i 0}{ }^{2}+y_{i 0}{ }^{2}\right) e^{j \Phi(0,0)}
$$

When $\exp [j \Phi(0,0\}] \doteqdot 1+j \Phi(0,0)$ and the magnitude of $\Phi(0,0)$ is small compared to unity,

$$
u_{\mathrm{c}}\left(x_{i 0}, y_{i 0}\right)^{\sim}-\frac{j}{2 \lambda^{3} Z_{1}^{2} Z_{i c}} e^{-j \frac{2 \pi}{\lambda} Z_{i c}} e^{-\frac{j \pi}{2 \lambda Z_{i c}}}\left(x_{i 0}{ }^{2}+y_{i 0}{ }^{2}\right)[1+j \Phi(0,0)]
$$

The magnification of $\left(j \pi / 2 \lambda Z_{\mathrm{ic}}\right)\left(x_{\mathrm{i} 0}^{2}+y_{\mathrm{i} 0}^{2}\right)$ is small compared to unity and we may also write

$$
u_{\mathrm{c}}\left(x_{i 0}, y_{i 0}\right)^{\sim} \frac{1}{\lambda^{2} Z_{1}^{2}} \frac{-j}{2 \lambda Z_{i c}} e^{-j \frac{2 \pi}{\lambda} Z_{i c}} e^{-\frac{j \pi}{2 \lambda Z_{i c}}}\left(x_{i 0}^{2}+y_{i 0}^{2}\right)[1+j \Phi(0,0)]
$$

Moreover, the complex amplitude of the zero-order term is expressed by $u_{0}\left(x_{\mathrm{i}}, y_{\mathrm{i}}\right)=\operatorname{Fr}\left[\left|r\left(x_{1}, y_{1}\right)\right|^{2}+\mid g\left(x_{1}\right.\right.$, $\left.y_{1}\right)\left.\right|^{2}$ ] and is given by

$$
u_{0}\left(x_{i}, y_{i}\right)=\frac{-1}{j \lambda Z_{i}} e^{\frac{-j 2 \pi}{\lambda} Z_{i}} \iint\left[\left|r\left(x_{1}, y_{1}\right)\right|^{2}+\left|\mathrm{g}\left(x_{1}, y_{1}\right)\right|^{2}\right] e^{-\frac{j n}{\lambda Z_{i}}\left[\left(x_{i}-x_{1}\right)^{2}+\left(y_{i}-y_{1}\right)^{2}\right]} d x_{1} d y_{1}
$$

$$
\begin{aligned}
u_{0}\left(x_{i 0}, y_{i 0}\right) & =\left(\frac{1}{\lambda^{2} Z_{1}^{2}}+\frac{1}{\lambda^{2} Z_{0}^{2}}\right) e^{\frac{-j 2 \pi Z_{i 0}}{\lambda}} \\
& =k_{0} e^{\frac{-j 2 \pi Z_{i 0}}{\lambda}}
\end{aligned}
$$

In these equations, $k_{0}=\left(1 / \lambda^{2} Z_{1}^{2}+1 / \lambda^{2} Z_{0}^{2}\right)$ is a constant. Three wavefronts of $u_{\mathrm{i}}\left(x_{\mathrm{i} 0}, y_{\mathrm{i} 0}\right), u_{\mathrm{c}}\left(x_{\mathrm{i} 0}, y_{\mathrm{i} 0}\right)$, and $u_{0}\left(x_{\mathrm{i} 0}, y_{\mathrm{i} 0}\right)$ exist in the image plane. To remove the zeroorder term, all complex amplitudes in the image plane are Fourier transformed, and the zero frequency is excluded; this means that $u_{0}\left(x_{\mathrm{i}}, y_{\mathrm{i}}\right)$ expressed by Eq. (14) is also removed. These complex amplitudes are then inverse Fourier transformed to the image plane. If we expand $\exp \left[-j \Phi\left(x_{\mathrm{i} 0} / M, y_{\mathrm{i} 0} / M\right)\right]$ with a Fourier series

$$
e^{-j \Phi\left(\frac{x_{i 0}}{\frac{M_{1} y_{i 0}}{M}}\right)}=\sum_{m=-\infty}^{m=\infty} c_{m} e^{j \frac{2 \pi m}{d} x_{i 0}},
$$

then $c_{0}=1$ and $c_{-\mathrm{m}}=-c_{\mathrm{m}}^{*},(m \neq 0)$. We Fourier transform the complex amplitudes of $u_{\mathrm{i}}\left(x_{\mathrm{i}}, y_{\mathrm{i}}\right)$ and $u_{\mathrm{c}}\left(x_{\mathrm{i}}, y_{\mathrm{i}}\right)$ again, and we add $\exp ( \pm j \pi / 2)= \pm j$ at the zero frequency. Eq. (7) and Eq. (14) then become: 


$$
\begin{aligned}
u_{i}\left(x_{i}, y_{i}\right)^{\sim} & \frac{1}{\lambda^{2} Z_{1}^{2}} e^{-\frac{j \pi}{\lambda} Z_{i 0}} e^{\frac{j \pi}{2 \lambda Z_{i 0}}}\left(x_{i}^{2}+y_{i}^{2}\right) e^{\frac{j \pi}{\lambda}\left(\frac{1}{2 Z_{i 0}}+\frac{1}{M^{2} Z_{0}}+\frac{1}{M^{2} Z}\right)\left(x_{i}^{2}+y_{i}^{2}\right)} \\
& \times\left[ \pm j-j \Phi\left(\frac{x_{i}}{M}, \frac{y_{i}}{M}\right)\right]
\end{aligned}
$$

$$
u_{c}\left(x_{i}, y_{i}\right)^{\sim} \frac{1}{\lambda^{2} Z_{1}^{2}} \frac{-j}{2 \lambda Z_{i c}} e^{-\frac{j 2 \pi}{\lambda} Z_{i c}} e^{-\frac{j \pi}{2 \lambda Z_{i c}}}\left(x_{i}^{2}+y_{i}^{2}\right)[ \pm j+j \Phi(0,0)]
$$

Using $Z_{\mathrm{ic}}=-Z_{\mathrm{i} 0}=Z Z_{1} / Z_{0}$, the intensity distribution $I_{\mathrm{i}}\left(x_{\mathrm{i} 0}, y_{\mathrm{i} 0}\right)$ in the image plane is obtained as

$$
\begin{aligned}
& I_{i}\left(x_{i 0}, y_{i 0}\right) \\
& =\left|u_{i}\left(x_{i 0}, y_{i 0}\right)+u_{c}\left(x_{i 0}, y_{i 0}\right)\right|^{2} \\
& =\left(\frac{1}{\lambda^{2} Z_{1}^{2}}\right)^{2} \mid\left[\begin{array}{c}
{\left[ \pm j-j \Phi\left(\frac{x_{i}}{M}, \frac{y_{i}}{M}\right)\right] e^{j \pi}\left\{\left(\frac{1}{\lambda}+\frac{1}{2 Z_{i 0}}+\frac{1}{M^{2} Z_{0}}+\frac{1}{M^{2} Z}\right)\left(x_{i 0}{ }^{2}+y_{i 0}{ }^{2}\right)+2 Z \frac{Z_{1}}{Z_{0}}\right\}} \\
-\frac{1}{2 \lambda Z_{i c}}[ \pm j+j \Phi(0,0)] e^{-\frac{j \pi}{\lambda}\left(2 Z \frac{Z_{1}}{Z_{0}}\right)}
\end{array} \mid\right.
\end{aligned}
$$

In Eq. (19), if $\lambda Z i c \gg 1+\Phi(0,0)$, the complex amplitude of the conjugate image can be neglected. The phase term of the complex amplitude of the reconstructed image is cancelled by calculation of the intensity, so that Eq. (19) becomes

$$
I_{i}\left(x_{i 0}, y_{i 0}\right)=k\left\{1 \pm 2 \Phi\left(\frac{x_{i 0}}{\frac{M, y_{i 0}}{M}}\right)\right\}
$$

where $k=\left\{1 /\left(\lambda^{2} Z_{1}^{2}\right)\right\}^{2}$ is a constant, and the term $\Phi^{2}$ has been neglected because of its smallness. We should note that Eq. (20) shows that if a Gabor hologram is recorded in the computer, it is possible to use Zernike's phase contrast observation method.

\section{Results}

The hard X-ray Gabor hologram is reconstructed by a collimated beam parallel to the axis, so the hologram intensity distribution is multiplied by unity. This means that the intensity distribution itself can be regarded as the complex amplitude and transformed from the hologram plane to the object plane. For this transformation, the Rayleigh-Sommerfeld diffraction formula was used. The image wavefront is produced at a long distance from the hologram.

The correct position determination in the image plane is important. A method to obtain an autofocused image has been proposed [18]. However, we will present a method to elegantly find the position in the image plane of simple objects recorded in the hologram, such as the polystyrene sphere beads. In our method, we use the diffraction effect that results from the distance between the image plane and the hologram being very long. The principle of the method is that if the object is located at the image plane, no diffraction pattern appears around the image; however, if the image is located at a position away from the image plane, a ring-shaped diffraction pattern appears around the image. Therefore, the position of the image plane can be elegantly determined by
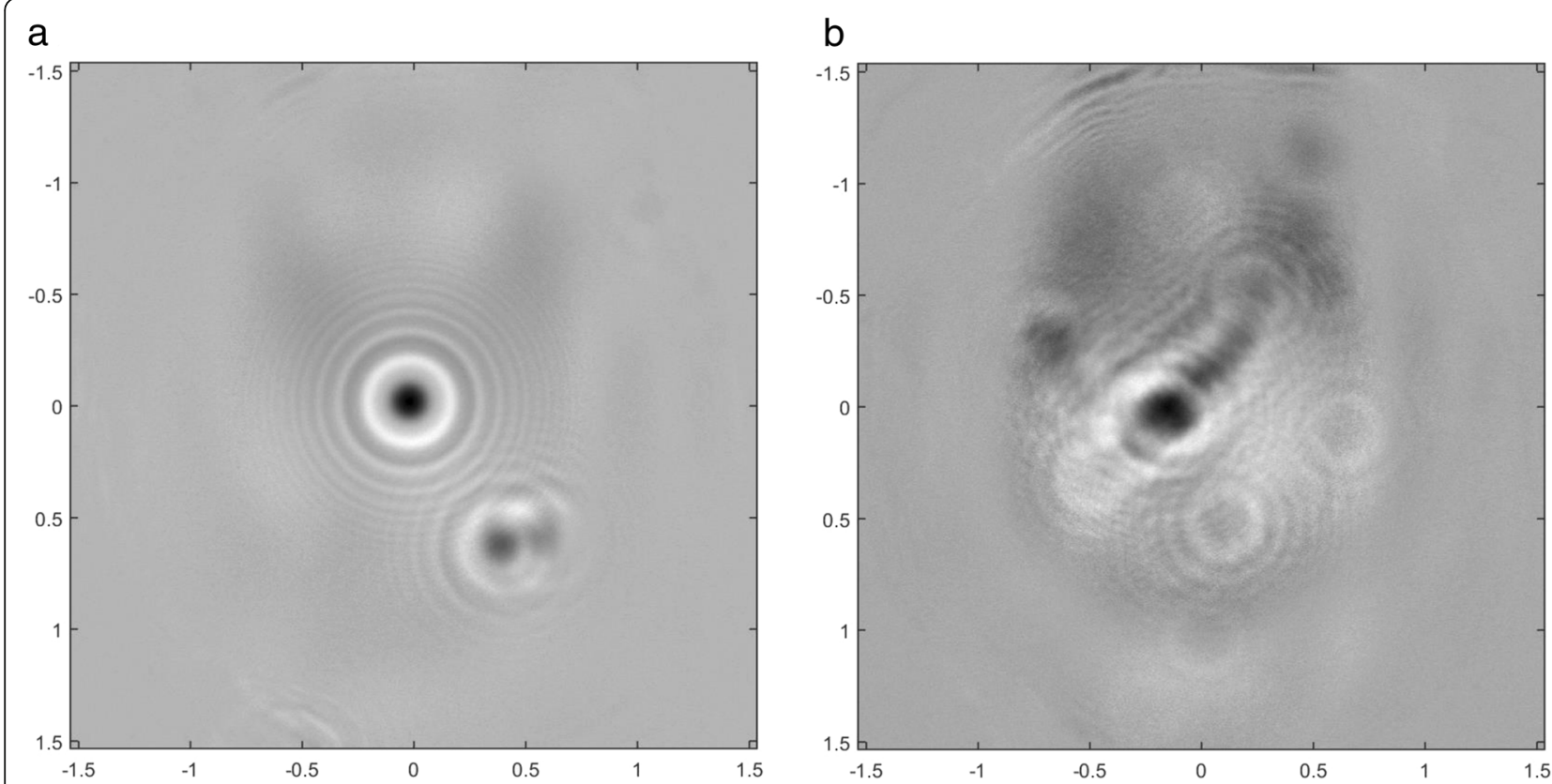

Fig. 4 Holograms constructed by hard X-ray, with $\lambda=0.1259 \mathrm{~nm}$ (X-ray energy of $9.85 \mathrm{keV}$ ). a Polystyrene sphere beads with $8 \mu \mathrm{m}$ diameter. $\mathbf{b}$ HeLa cells (human cells) 

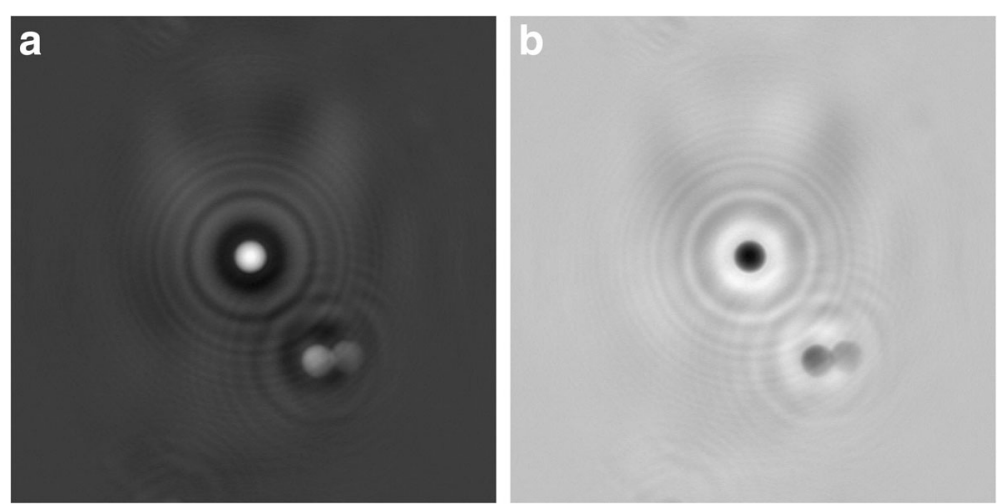

Fig. 5 Experimental results of Zernike's phase contrast method using polystyrene sphere beads with $8 \mu m$ diameter. a $l_{i}\left(x_{i}, y_{i}\right)=k\left\{1-2 \Phi\left(x_{i 0} / M\right.\right.$, $\left.\left.y_{\mathrm{i} 0} / M\right)\right\} . \mathbf{b} k\left\{1+2 \Phi\left(x_{\mathrm{iO}} / M, y_{\mathrm{iO}} / M\right)\right\}$

observing the diffraction patterns (the image plane $Z_{\mathrm{i} 0}$ was $174.5 \mathrm{~m}$ ). The magnification $M$ of the optical system [19] was calculated to be $M=\left|Z_{\mathrm{i} 0} / Z\right|=25.55$, given that the distance between object and hologram was $Z=$ $6.830 \mathrm{~m}$.

Figure 4a and $\mathrm{b}$ show the hard X-ray Gabor holograms of the polystyrene beads and the HeLa cells, respectively. Since the wavefront produced by the X-rays transmitted through the phase object is advanced in comparison with the wavefront traveling in the air, the hologram becomes dark. Figures 5 and 6 show the results of Zernike's phase contrast method. Figure 5 shows the results obtained with the $8-\mu \mathrm{m}$ polystyrene sphere bead samples. Figure 5a shows $I_{\mathrm{i}}\left(x_{\mathrm{i}}, y_{\mathrm{i}}\right)=k\left\{1-2 \Phi\left(x_{\mathrm{i} 0} / M, y_{\mathrm{i} 0} / M\right)\right\}$, and Fig. 5b shows $k\left\{1+2 \Phi\left(x_{\mathrm{i} 0} / M, y_{\mathrm{i} 0} / M\right)\right\}$ as noted in Eq. (20). The reconstructed phase object is the bright sphere in Fig. 5a and the dark sphere in Fig. 5b. Figure 6 shows the results obtained for the dried HeLa cell samples. Figure 6a shows $I_{\mathrm{i}}\left(x_{\mathrm{i}}, y_{\mathrm{i}}\right)=k\left\{1-2 \Phi\left(x_{\mathrm{i} 0} / M, y_{\mathrm{i} 0} / M\right)\right\}$, and Fig. 6b shows $k\left\{1+2 \Phi\left(x_{\mathrm{i} 0} / M, y_{\mathrm{i} 0} / M\right)\right\}$. It should be pointed out that $\left.\Phi\left(x_{\mathrm{i} 0} / M, y_{\mathrm{i} 0} / M\right)\right\}$ is negative, because with hard X-rays the object refractive index is smaller than that of air.

\section{Discussion}

It should be noted that the refractive index related to the phase difference in the hard X-ray regime is less than unity, contrary to what happens with visible light; the well-known refractive index equation is approximately given by $n=1-1.35 \times 10^{-6} \rho \lambda^{2}$, where $\rho\left(\mathrm{g} / \mathrm{cm}^{3}\right)$ is the density, $\lambda(\AA)$ is the wavelength [12], and the terms representing absorption and scattering are ignored for simplicity. The maximum value of the optical path difference in the polystyrene sphere is about $\delta=0.017 \mathrm{~nm}$, if the density is taken as $\rho \doteqdot 1$. The condition needed to derive Eq. (20) is $\Phi\left(x_{\mathrm{i}} / M, y_{\mathrm{i}} / M\right)<1$; the magnitude of $\Phi\left(x_{\mathrm{i}} / M, y_{\mathrm{i}} / M\right)=2 \pi \delta / \lambda$ recorded in the hologram is about 0.848 . Since this value is smaller than unity, the condition is satisfied [4]. In Eq. (10), a value of $\left(4\left(0.82 \lambda Z_{i c}\right) / M\right)^{1 / 2}=53 \mu \mathrm{m}$ in the object plane was obtained, so that the wavefront of the conjugate image in the image plane can be regarded as a wavefront caused from a point source. Samples of polystyrene sphere beads with $8-\mu \mathrm{m}$ diameter and dried HeLa cells are used as phase objects; in the used hologram, the aperture diameter magnitude can be considered to produce a wavefront originating from a point source. The
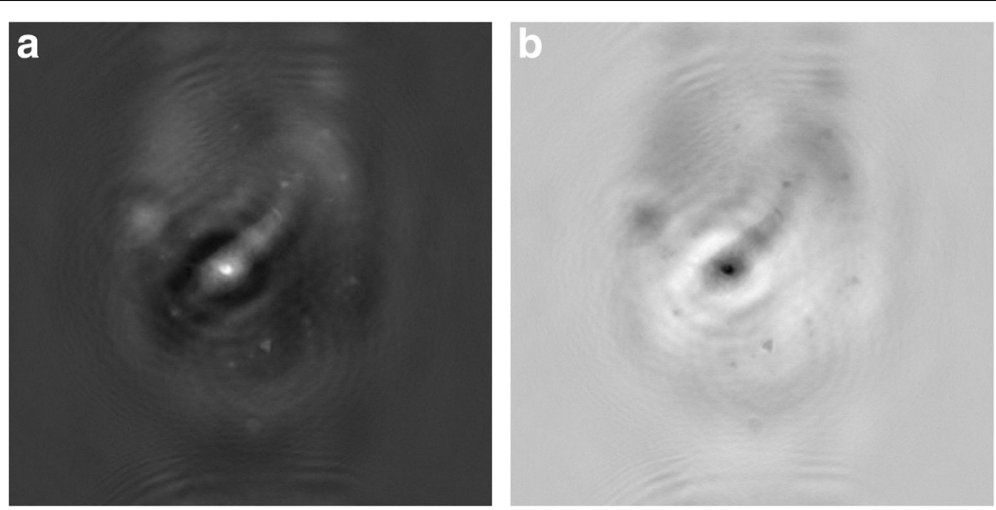

Fig. 6 Experimental results of Zernike's phase contrast method using HeLa cells. a $l_{i}\left(x_{i}, y_{i}\right)=k\left\{1-2 \Phi\left(x_{i 0} / M, y_{i 0} / M\right)\right\} . \mathbf{b} k\left\{1+2 \Phi\left(x_{i 0} / M, y_{i 0} / M\right)\right\}$ 
numerical value of $(1+\Phi(0,0)) /\left(2 \lambda Z_{\text {ic }}\right)$ in Eq. (20) is calculated, to check whether this term can be neglected or not, resulting in $\Phi(0,0)<1, \lambda=0.1259 \times 10^{-3} \mu \mathrm{m}$, and $Z_{\text {ic }}=174.5 \times 10^{6} \mu \mathrm{m}$; therefore, $(1+\Phi(0,0)) /\left(2 \lambda Z_{\text {ic }}\right) \sim$ $4.2 \times 10^{-5} \ll 1$, and we can safely neglect this term.

\section{Conclusions}

We proposed a microscope using Zernike's phase contrast observation method and a hard X-ray Gabor hologram recorded in a computer. Two different sample types were used for demonstration purposes: polystyrene sphere beads with an 8- $\mu \mathrm{m}$ diameter, and dried HeLa cells. Recording the hologram in a computer makes it possible to perform computational experiments on coherent X-ray processing. This is very important, because $\mathrm{X}$-ray resources are limited. Moreover, coherent numerical processing becomes easy and simple; creating a perfect phase delay of $\pi / 2$, for example, is trivially produced numerically. Even though a small $0.1259 \mathrm{~nm}$ wavelength was used, there is no influence of the external vibrations in image reconstruction. It is also pointed out that the use of holography with the exception of an image hologram can produce images with high resolution, since the pixel size of the CCD detector does not have influence on resolution, but its diameter has.

\section{Abbreviations}

CCD: Charge coupled device; DIC: Differential interference contrast;

FZP: Fresnel zone plate; NA: Numerical aperture; $\lambda$ : Wavelength

\section{Acknowledgements}

The authors would like to thank Dr. Y. Koseki, a group leader, and Dr. K. Chinzei, a sub-director of AIST, Japan, for providing research facilities. Technical advices from Dr. K. Hibino, Dr. T. Eijyu and Dr. M. Yamauchi were highly appreciated.

\section{Funding}

Consejo Nacional de Ciencia y Tecnología(CONACYT), Mexico, has awarded to Dr. Juan C. Aguilar with a scholarship to make a postdoctoral research at National Institute of Advanced Industrial Science and Technology (AIST), Japan (250204). A part of this study was supported by 2016 Saga Prefecture Leading Industry Incubation Program, Japan.

\section{Availability of data and materials}

The datasets supporting the conclusions of this article are included within the present article.

\section{Authors' contributions}

KM: He engaged in the idea of the method, mathematical analysis, simulation of laser and X-ray holography and design of computer algorithm. JCA: He engaged in a computer code for hard X-ray holography. MM: He engaged in the management of this study, discussion and advice of X-ray measurement method. MY: He engaged in X-ray optical design, production of hard X-ray hologram and advice for simulation of laser holography. SR: He engaged in a part of the idea of the method, simulation of laser holography and computer algorithm. YS: He engaged in production of hard X-ray hologram. AT: He engaged in production of hard X-ray hologram. IN: He engaged in discussion of hard X-ray optics and simulation of laser holography. All authors read and approved the final manuscript.

\section{Ethics approval and consent to participate}

This study does not need any ethical approval along with evidence. The samples used in this study are commercially distributed.

\section{Author details}

${ }^{1}$ Theranostic Device Research Group, Health Research Institute, National Institute of Advanced Industrial Science and Technology (AIST), 1-2-1 Namiki, Tsukuba, Ibaraki 305-8564, Japan. ${ }^{2}$ The graduate School for the Creation of New Photonics Industries, 1955-1 Kurematsu, Nishi-ku, Hamamatsu, Shizuoka 431-1202, Japan. Instituto Nacional de Astrofísica," Óptica y Electrónica, Luis Enrique Erro \#1, Tonantzintla, Puebla, Mexico. ${ }^{4}$ Research Institute for Measurement and Analytical Instrumentation, NMIJ, National Institute of Advance Industrial Science and Technology, Tsukuba 305-8568, Ibaraki, Japan. ${ }^{5}$ Japan Synchrotron Radiation Research Institute, SPring-8, Sayo, Hyogo 679-5198, Japan. ${ }^{6}$ Singapore-MIT Alliance for Research and Technology (SMART) Centre1 CREATE Way \#09-03, CREATE Tower, Singapore 138602, Singapore. ${ }^{7}$ Faculty of Technology, University of Oulu, PO Box 73009014 Oulu, Finland.

Received: 25 August 2016 Accepted: 7 November 2016 Published online: 23 November 2016

\section{References}

1. Suzuki, Y, Takeuchi, A: "Reduction of Speckle Noises by Spatial Filter Method in Hard X-ray Region", AIP Conf. Proc. 1234, p. 453 (2010). (http://scitation.aip.org/ content/aip/proceeding/aipcp/10.1063/1.3463238. Accessed 15 Nov 2016)

2. Momose, A., Takeda, T., Tani, Y., Hirano, K.: Phase-contrast X-ray composed tomography for observing biological soft tissues. Nat. Med. 2, 473-475 (1996)

3. $\mathrm{Xu}, \mathrm{W}$. , Jericho, M.H., Meinertzhagen, I.A., Kreuzer, H.J.: Digital in-line holography for biological application, pp. 11301-11305. (2001). PNAS98

4. Born, M, Wolf, E: Principles of Optics 2nd Ed, pp. 424-425, and p. 524. London, Pergamon Press Ltd. (1991)

5. Momose, A.: Demonstration of phase-contrast $X$-ray computed tomography using an X-ray interferometer. Nucl. Instrum. Methods. A352, 622-628 (1995)

6. Momose, A, Fujii, A, Kadowaki, H, Jinnai, H: Three dimensional observation of polymer blend by X-ray phase tomography. Macromolecules. 38, (2005) pp. 622-628

7. Matsuda, K, Rehman, S, Oohashi, H, Niskanen, I, Yamauchi, M, Nakatani, T, Homma, $\mathrm{K}$, and Peiponen, $\mathrm{K}$ : Digital holographic microscopy using a single mode fiber, Proc. The Eighth Finland-Japan Joint Symposium on Optics in Engineering, 3-5 September 2009, Technical Digest, Tokyo, Japan, pp. 17-19 (2009)

8. Matsuda, K., Namiki, M.: Holographic lateral shear interferometer for differential interference contrast method. J. Optics. (Paris) 11, $81-85$ (1980)

9. Goodman, JW: Introduction to Fourier Optics, 2nd Ed, p.157 and p.74. New York, McGraw Hill Co. Inc. (1988)

10. Gabor, D.: A new microscopic principle. Nature 161, 777 (1948)

11. Suzuki, Y., Takeuchi, A.: Gabor holography with speckle-free spherical wave in hard X-ray region. Jpn. J. Appl. Phys. 51, 086701-1 (2012)

12. Matsuda, K, Rehman, S, Lopez, JCA, Suzuki, Y, Takeuchi, A, Misawa, M, Niskanen, I: Phase contrast microscope using a hard X-ray Gaborhologram, Proc. The Eleventh Finland-Japan Joint Symposium on Optics in Engineering, 1-3 September 2015, Joensuu, Finland, p. 6-7 (2015)

13. Chapman, H.N., Nugent, K.A.: Coherent lensless X-ray imaging. Nat. Photonics 4, 833-839 (2010)

14. Rodenburg, J.M., Hurst, A.C., Cullis, A.G., Dobson, B.R., Pfeiffer, F., Bunk, O., David, C., Jefimovs, K., Johnson, I.: Hard X-ray lensless imaging of extended objects. Phys. Rev. Letts 98, 034801 (2007). 1-4

15. Snigirev, A., Snigireva, I., Kohn, V., Kuznetsov, S., Schelokov, I.: On the possibilities of X-ray phase contrast microimaging by coherent high-energy Synchrotron radiation. Rev. Sci. Instrum. 66, 5486-5492 (1995)

16. Wilkins, S.W., Gureyev, T.E., Gao, D., Pogany, A., Stevenson, A.W.: Phase contrast imaging using polychromatic hard X-ray. Nature 384, 335-338 (1996)

17. Latychevskaia, T., Fink, H.W.: Solution to the twin image problem in holography. Phy. Rev. Lett 98, 233901 (2007). 1-4

18. Langehanenberg, P., Kemper, B., Dirksen, D., Von Bally, G.: Autofocusing in digital holographic phase contrast microscopy on pure phase objects for live cell imaging. Appl Optics. 47, D176-D182 (2008)

19. Meier, R.W.: Magnification and third-order aberrations in holography. J. Opt. Soc. Am. 55, 46-51 (1965) 